\title{
High prevalence of undiagnosed diabetes mellitus in Southern Germany: Target populations for efficient screening. The KORA survey 2000
}

\author{
W. Rathmann ${ }^{1}$, B. Haastert ${ }^{1}$, A. Icks ${ }^{1}$, H. Löwel ${ }^{2}$, C. Meisinger ${ }^{2}$, R. Holle ${ }^{2}$, G. Giani ${ }^{1}$ \\ ${ }^{1}$ Department of Biometrics and Epidemiology, German Diabetes Research Institute at Düsseldorf University, Düsseldorf, Germany \\ ${ }^{2}$ GSF, Neuherberg, Germany
}

\section{Abstract}

Aims/hypothesis. To estimate the prevalence of undiagnosed diabetes mellitus, impaired glucose tolerance (IGT) and impaired fasting glucose (IFG), and their relations with cardiovascular risk factors in the general population aged 55 to 74 years in Southern Germany.

Methods. Oral glucose tolerance tests were carried out in a random sample of 1353 subjects aged 55 to 74 years participating in the KORA (Cooperative Health Research in the Region of Augsburg) Survey 2000. Prevalences of glucose tolerance categories (1999 WHO criteria) were adjusted for sample probabilities. The numbers needed to screen (NNTS) to identify one person with undiagnosed diabetes were estimated from age-adjusted logistic regression models.

Results. Sample design-based prevalences of known and unknown diabetes, IGT, and IFG were 9.0\%, $9.7 \%, 16.8 \%, 9.8 \%$ in men, and $7.9 \%, 6.9 \%, 16.0 \%$, $4.5 \%$ in women, respectively. In both sexes, participants with undiagnosed diabetes had higher BMI, waist circumference, systolic blood pressure, triglyce- rides, uric acid, and lower HDL-cholesterol than normoglycaemic subjects. A combination of abdominal adiposity, hypertension, and parental diabetes in men resulted in a NNTS of 2.9 (95\%CI: 2.0-4.6). In women, the combination of increased triglycerides, hypertension and parental diabetes history yielded a NNTS of 3.2 (95\% CI: 2.2-5.1).

Conclusion/interpretation. About $40 \%$ of the population aged 55 to 74 years in the Augsburg region have disturbed glucose tolerance or diabetes. Half of the total cases with diabetes are undiagnosed. Cardiovascular risk factors worsen among glucose tolerance categories, indicating the need for screening and prevention. Screening for undiagnosed diabetes could be most efficient in individuals with abdominal adiposity (men), hypertriglyceridaemia (women), hypertension, and parental diabetes history. [Diabetologia (2003) 46:182-189]

Keywords Undiagnosed diabetes, impaired glucose tolerance, epidemiology, cardiovascular risk, triglycerides, obesity, screening, public health.
Received: 10 September 2002 / Revised: 17 October 2002 Published online: 18 February 2003

CC Springer-Verlag 2003

Corresponding author: Dr. W. Rathmann MSPH (USA), Dept. of Biometrics and Epidemiology, German Diabetes Research Institute at Düsseldorf University, Auf'm Hennekamp 65, 40225 Düsseldorf, Germany

E-mail: rathmann@ddfi.uni-duesseldorf.de

Abbreviations: KORA, Kooperative Gesundheitsforschung im Raum Augsburg (Cooperative Health Research in the Region of Augsburg); WHO MONICA, Monitoring of Trends and Determinants in Cardiovascular Disease project; NNTS, number needed to screen.
Surveillance of diabetes is a necessary first step towards its prevention and control [1], to reduce the increased risk of cardiovascular morbidity and mortality [2]. In Europe, population-based epidemiologic studies on prevalence of known and undiagnosed diabetes mellitus using oral glucose tolerance tests (OGTT) are still rare [3]. Therefore, a mapping and comparative analysis of the prevalence of Type 2 diabetes and impaired glucose tolerance (IGT) in Europe, which has been successfully carried out for Type 1 diabetes [4], is lacking.

In Germany, the country with the largest population in Europe, there are only data on self-reported diabe- 
tes, e.g. from the National Health Survey and the MONICA Augsburg project [5, 6]. Population-based data on diabetes prevalence (diagnosed and undiagnosed) using OGTT are lacking, which would allow comparisons with similar studies from other European countries $[3,7]$. Due to intensive health care utilization by the population, it has been hypothesized that the frequency of undiagnosed diabetes in Germany is low [5].

As part of the KORA (Cooperative Health Research in the Region of Augsburg) Survey 2000, fasting OGTT were carried out among participants aged 55 to 74 years without known diabetes. The KORA Survey is a population-based study in Southern Germany using the same geographical region and study methods as the former WHO MONICA project [8]. This paper describes the age-sex-specific prevalences of newly diagnosed diabetes (1999 WHO criteria), IGT, and impaired fasting glucose (IFG) in this population, and their associations with cardiovascular risk factors. Little is known about the risk factors associated with undiagnosed diabetes. Therefore, potential risk factors for undiagnosed diabetes and the number needed to screen in risk-groups were assessed to detect target populations for efficient screening.

\section{Subjects, Materials and Methods}

Study population. Subjects were invited to participate from the city of Augsburg and 16 towns and villages out of 70 communities from the surrounding districts with about 600000 inhabitants in 1999. The survey sampling method of the former WHO MONICA project was used [9]. Within each selected community, a stratified sample with ten equal strata by sex and age was drawn, four of these strata included men and women aged 55 to 74 years.

The total study sample involved 6640 subjects with 2656 aged 55 years or over. After an intensive media campaign in the region, all subjects in the age-group 55 to 74 years without known diabetes were invited to participate in the OGTT study and informed consent was obtained from the participants. The study was performed from October 1999 to April 2001 in the KORA study centre (city of Augsburg) and 11 local study centres in the communities. All subjects who refused to take part were asked for a short structured telephone interview to obtain information on their medical conditions (including known diabetes and obesity).

Oral glucose tolerance tests. OGTT were carried out in the morning (7:00 am to 11:00 am). Participants were asked to fast for $10 \mathrm{~h}$ overnight, to avoid heavy physical activity on the day before examination and to refrain from smoking before and during the test. Exclusion criteria for the OGTT were: (i) antidiabetic medication or known diabetes, eventually confirmed by the patient's general practitioner; (ii) acute illness (infection, fever, acute gastrointestinal disease); (iii) start of the OGTT later than 11:00 am.

Fasting venous blood glucose was sampled and $75 \mathrm{~g}$ of anhydrous glucose given (Dextro OGT, Boehringer Mannheim, Germany). The means \pm SD duration for the 2-h glucose determination among all KORA OGTT participants was $120 \pm 6$ min.

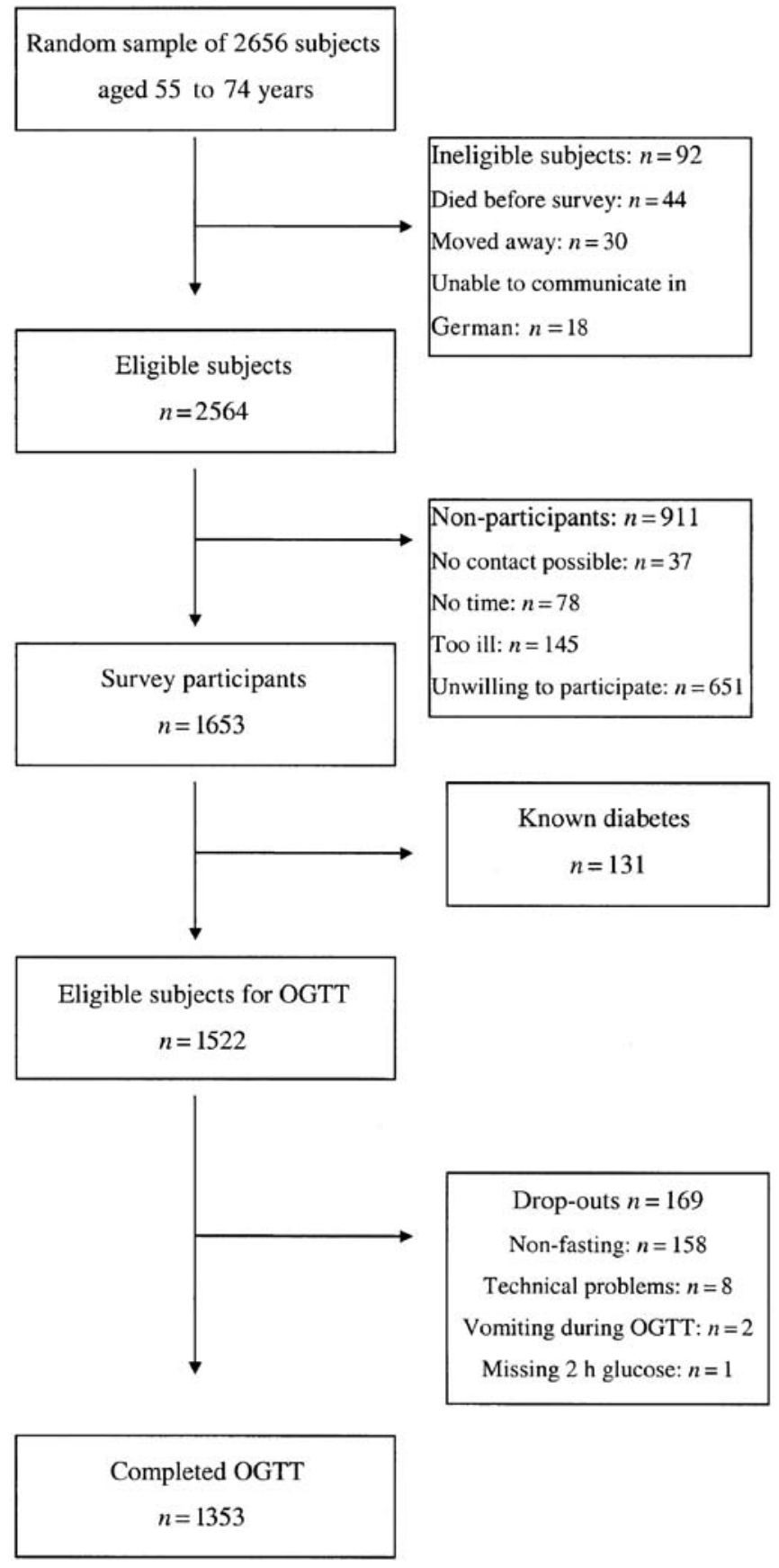

Fig. 1. Flowchart of participation in the KORA Survey 2000 OGTT examination

Subjects with gastrointestinal disturbances (vomiting, diarrhoea) after the glucose load were excluded from the analyses (Fig. 1).

Previously known diabetes was defined based on self-reported physician diagnosis or use of antidiabetic agents. Newly diagnosed diabetes $(\geq 7.0 \mathrm{mmol} / \mathrm{l}$ fasting or $\geq 11.1 \mathrm{mmol} / \mathrm{l} 2-\mathrm{h}$ post glucose load), IGT, IFG, and normal glucose tolerance (NGT) were defined according to the 1999 WHO diagnostic criteria based on both fasting and postchallenge glucose values [10]. Furthermore, prevalence of undiagnosed diabetes was also estimated based on a fasting value of more than or equal to $7.0 \mathrm{mmol} / \mathrm{l}$, as proposed by the American Diabetes Association (ADA) [11]. 
Laboratory measurements. Blood was collected with minimal stasis, refrigerated at 4 to $8^{\circ} \mathrm{C}$ and shipped on refrigerant packaging within 2 to $4 \mathrm{~h}$ to the laboratory of Augsburg Central Hospital. Blood glucose was measured using a hexokinase method (Gluco-quant; Roche Diagnostics, Mannheim, Germany). The interassay coefficients of variation for glucose were $2.4 \%$ at $98 \mathrm{mg} / \mathrm{dl}$ and $2.1 \%$ at $235 \mathrm{mg} / \mathrm{dl}$. $\mathrm{HbA}_{1 \mathrm{c}}$-values were assessed using a turbidimetric immunologic assay (Tina-quant, Roche Diagnostics). The interassay coefficients of variation were $3.9 \%$ at $\mathrm{HbA}_{1 \mathrm{c}} 5.7 \%$ and $5.2 \%$ at $\mathrm{HbA}_{1 \mathrm{c}} 9.7 \%$.

Total cholesterol was measured using the Boehringer CHOD-PAP (Roche Diagnostics) and HDL-cholesterol using the phosphotungstic acid method (Boehringer Mannheim). Low HDL-cholesterol was defined as less than $0.91 \mathrm{mmol} / \mathrm{l}$ in men and less than $1.16 \mathrm{mmol} / \mathrm{l}$ in women. Triglycerides were measured with the Boehringer GPO-PAP assay (nonfasting in diabetic subjects). Hypertriglyceridaemia was defined as concentrations more than or equal to $2.0 \mathrm{mmol} / \mathrm{l}$. Serum uric acid was assessed with the uricase method (Roche Diagnostics). Hyperuricaemia was defined as concentrations more than $416 \mu \mathrm{mol} / \mathrm{l}$ in men and more than $357 \mu \mathrm{mol} / \mathrm{l}$ in women.

Anthropometric measurements and interviews. Body weight was measured in light clothing by trained investigators to the nearest $0.1 \mathrm{~kg}$, and height to the nearest $0.5 \mathrm{~cm}$. Waist circumference was measured at the minimum abdominal girth and hip circumference was assessed at the maximum protusion of the hips at the level of the symphysis pubis to the nearest $0.1 \mathrm{~cm}$. Obesity was defined as BMI more than or equal to $30 \mathrm{~kg} / \mathrm{m}^{2}$ and abdominal adiposity as waist circumference above the 80th sex-specific centile (men: $>109 \mathrm{~cm}$; women: $>100 \mathrm{~cm}$ ) [12].

Blood pressure was measured three times at the right arm in a sitting position after a fifteen-minute rest using a validated automatic device (OMRON HEM 705-CP). The mean of the second and third measurement was used for the analysis. Hypertensive blood pressure was defined by systolic blood pressure more than $140 \mathrm{~mm} \mathrm{Hg}$ or diastolic blood pressure more than $90 \mathrm{~mm} \mathrm{Hg}$ (with or without antihypertensive medications).

Medical history was assessed in a structured interview including use of prescription drugs. Subjects were also asked to report the frequency and average duration of regular moderate and vigorous physical activity during leisure time in winter and summer using four categories. Responses for the two seasons were combined to define physical activity. Less than $1 \mathrm{~h}$ activity per week was defined as low physical activity. Parental history of diabetes was also assessed and was defined as either maternal or paternal diabetes or both.

Statistical analysis. Analyses were carried out separately for men and women. Age-specific prevalences (95\% confidence intervals) of diabetes (known and newly diagnosed), IGT, and IFG were calculated accounting for sample design using Stata Statistical Software: Release 7.0 (StataCorp, 2001, College Station, Tex., USA). Analyses were accounted for sampling weights and clustering to obtain point estimates, standard deviations and 95\% confidence intervals [13, 14]. The sampling weight for each individual was computed as the inverse of the product of the selection probability of the cluster and the proportion of responders in the sex-age-strata of the cluster. Therefore, some weighting adjustment was done for nonresponse [13]. Sample design-based standard deviations were computed from the standard errors [15]. Sample design-based trend tests (age) for the various glucose tolerance categories were carried out by including an ordinal variable (four ageclasses) in a logistic regression model. Crude age-sex-specific prevalences of known diabetes, new diabetes, IGT, and IFG were also directly standardized to the German population (December 31, 2000).

Sample design-based linear and logistic regression models were fitted to assess differences between groups for normaldistributed or dichotomous variables. Geometric means and standard deviation factors were computed for log-normal distributed variables (triglycerides).

Potential risk factors for newly diagnosed diabetes were evaluated using sample design-based logistic regression models with OGTT-based nondiabetic subjects as reference category. The number needed to screen (NNTS) to identify one subject with undiagnosed diabetes was calculated for various riskfactor-groups [16]. NNTS $(95 \% \mathrm{CI})$ were obtained from sample design-based logistic regression models as the inverse of the estimated prevalence of undiagnosed diabetes in the riskgroups. Finally, differences between participants and nonparticipants were assessed using Chi-square tests or Student's $t$ test, and by fitting a multiple logistic regression model with non-participation as dependent variable. A $p$ value of less than 0.05 was considered statistically significant.

\section{Results}

Characteristics of participants and non-participants. Overall, $1653(62 \%)$ of 2656 randomly selected subjects in the age-group 55 to 74 years participated in the survey (Fig. 1). Compared to participants, nonparticipants were older (participants: 47\% aged 65-74 years, non-participants: $56 \%$ aged $65-74$ years) $(p<0.001)$. There was no difference with respect to sex between the groups (women: $49 \%$ vs $52 \%)(p=0.22)$. After excluding participants with known diabetes and further drop-outs, which were mainly subjects who were not able to attend an investigation during the morning hours $(n=158), 1353$ subjects had a standard OGTT (Fig. 1).

Prevalence of diabetes and glucose intolerance. The total sample design-based diabetes prevalence (diagnosed and undetected cases) in the age-group 55 to 74 years was $16.6 \%$ (Table 1). The age-standardized (German 2000 population) prevalences for the various glucose tolerance categories were comparable to the sample design-based Augsburg estimates. About half of all cases with diabetes were undiagnosed, which varied little across age. Overall, previously unknown diabetes was more frequently found among men than women (Table 1).

According to the fasting ADA criteria, sample design-based prevalence of undiagnosed diabetes was 4.9\% (95\% CI: 3.7-6.1\%) (men: 6.4\%; women: $3.5 \%$ ). Thus, $41 \%$ of newly diagnosed cases were detected by 2-h glucose only (men: $34 \%$; women: $50 \%$ ).

IFG was about two times more common in men than in women $(9.8 \%$ vs $4.5 \%)(p<0.01)$, whereas no sex-difference was observed for IGT $(p=0.70)$ (Table 1). Prevalence of IGT increased with age in both sexes (both $p<0.01$ ), affecting about one-fifth of participants more than or equal to 70 years of age. For IFG, an in- 
Table 1. Sample design-based and age-standardized prevalences of known and newly diagnosed diabetes mellitus (1999 WHO criteria) and other categories of hyperglycaemia: the KORA Survey 2000, Augsburg

\begin{tabular}{|c|c|c|c|c|c|c|}
\hline $\begin{array}{l}\text { Age-sex- } \\
\text { groups }\end{array}$ & $\begin{array}{l}\text { Study } \\
\text { population } \\
(n)\end{array}$ & $\begin{array}{l}\text { Known } \\
\text { diabetes } \\
(\%)\end{array}$ & $\begin{array}{l}\text { Newly } \\
\text { diagnosed } \\
\text { diabetes } \\
(\%)\end{array}$ & $\begin{array}{l}\text { Impaired } \\
\text { glucose } \\
\text { tolerance } \\
(\%)\end{array}$ & $\begin{array}{l}\text { Impaired } \\
\text { fasting } \\
\text { glucose } \\
(\%)\end{array}$ & $\begin{array}{l}\text { Normal } \\
\text { glucose } \\
\text { tolerance } \\
(\%)\end{array}$ \\
\hline \multicolumn{7}{|l|}{ Men: } \\
\hline $55-59$ yrs & 194 & 7.2 & 9.5 & 8.6 & 9.5 & 65.2 \\
\hline $60-64$ yrs & 210 & 7.2 & 8.1 & 18.8 & 12.9 & 53.0 \\
\hline 55-74 yrs: & 770 & & & & & \\
\hline sample-based & & 9.0 & 9.7 & 16.8 & 9.8 & 54.8 \\
\hline age-standardized & & 9.3 & 9.3 & 17.0 & 9.9 & 54.5 \\
\hline \multicolumn{7}{|l|}{ Women: } \\
\hline 55-74 yrs: & 715 & & & & & \\
\hline sample-based & & 7.9 & 6.9 & 16.0 & 4.5 & 64.7 \\
\hline age-standardized & & 8.0 & 6.9 & 15.7 & 4.4 & 65.0 \\
\hline Total $(95 \% C I):$ & 1485 & & & & & \\
\hline sample-based & & $8.4(7.3-9.5)$ & $8.2(6.7-9.6)$ & $16.4(13.8-18.9)$ & $7.0(5.9-8.1)$ & $60.1(56.7-63.4)$ \\
\hline age-standardized ${ }^{\mathrm{a}}$ & & $8.7(7.3-10.1)$ & $8.2(6.8-9.6)$ & $16.4(14.5-18.3)$ & $7.2(5.9-8.5)$ & $59.5(57.0-62.0)$ \\
\hline
\end{tabular}

CI: confidence interval aGerman population (31.12.2000)

crease with age was only found in women $(p<0.05)$ (men: $p=0.09$ ) (Table 1).

Thus, about $40 \%$ of the total population in the agegroup 55 to 74 years in the Augsburg region either had diabetes or other categories of glucose intolerance. The total prevalence of both diagnosed and undiagnosed glucose disorders in men exceeded that among women (men: 45\%; women: $35 \%)(p<0.001)$.

Characteristics of subjects with known and newly diagnosed diabetes. Among patients with previously known diabetes (mean age: $64.9 \pm 4.9$ years; men: $50 \%$; diabetes duration: $9.7 \pm 7.8$ years); $57 \%$ used oral antidiabetic drugs only and $33 \%$ were treated with insulin, including combinations with oral agents. Average $\mathrm{HbA}_{1 \mathrm{c}}$ value $( \pm \mathrm{SD})$ in patients with known diabetes was $7.2 \pm 1.4 \%$ (116\% of the upper limit of normal range). In participants with newly diagnosed diabetes (mean age: $64.6 \pm 5.4$; men: $56 \%$ ), the mean $\mathrm{HbA}_{1 \mathrm{c}}$ of $6.2 \pm 1.0 \%$ was intermediate between known diabetes and NGT $(5.6 \pm 0.3 \%)$ and was higher compared to both IGT $(5.7 \pm 0.4 \%)$ and IFG $(5.6 \pm 0.3 \%)$ (both $p<0.001)$.

The proportions of subjects with more than one visit to a physician during the last 4 weeks were $64 \%$, $35 \%$, and $41 \%$ in men, and $65 \%, 39 \%$, and $47 \%$ in women with known, previously undiagnosed diabetes, and NGT, respectively, which was increased in patients with known diabetes only $(p<0.05)$. The corresponding proportions for more than one hospitaliza- tion during the preceding 12 months among those groups were $23 \%, 21 \%, 15 \%$ in men and $24 \%, 26 \%$, $14 \%$ in women, respectively, which were also higher only in known diabetes compared to NGT $(p<0.05)$.

Cardiovascular risk factors and undiagnosed diabetes. In both sexes, subjects with newly diagnosed diabetes had an increased cardiovascular risk factor profile, which resembled the characteristics of known diabetic patients (Table 2). In general, participants with undiagnosed diabetes had higher BMI, waist circumference, systolic blood pressure, triglycerides, serum uric acid, and lower HDL-cholesterol than normoglycaemic participants. Prevalence of obesity (BMI $\geq 30 \mathrm{~kg} / \mathrm{m}^{2}$ ) was $39 \%$ in men with undetected diabetes (NGT: 18\%), which was even higher in women (undetected diabetes: $46 \%$; NGT: $27 \%$ ). In participants with IGT and IFG, most cardiovascular risk factors were intermediate between known diabetes and normal glucose tolerance (Table 2).

Number needed to screen (NNTS) for undiagnosed diabetes. The association of known risk factors for Type 2 diabetes and metabolic parameters with undiagnosed diabetes was evaluated, all could be assessed in primary care, in order to identify subgroups for most efficient screening (low NNTS).

Undiagnosed diabetes was associated with abdominal adiposity, hypertensive blood pressure, hyper- 
Table 2. Sample design-based cardiovascular risk factors in 1485 survey participants aged 55 to 74 years by glucose tolerance categories: the KORA Survey 2000, Augsburg, Germany

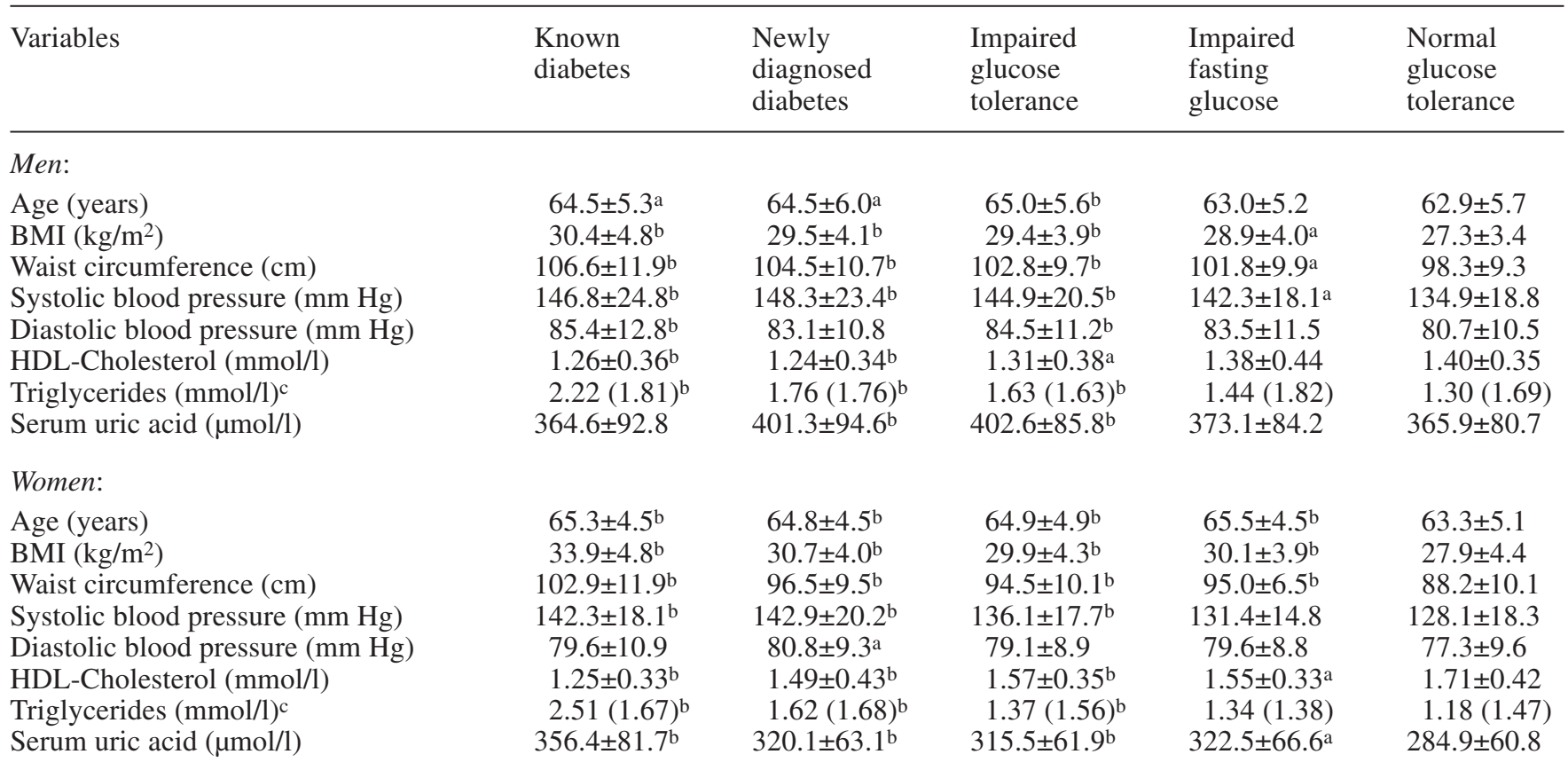

Data are means \pm SD or geometric mean (SDF) (triglycerides)

${ }^{\mathrm{a}} p<0.05,{ }^{\mathrm{b}} p<0.01$ : age- and sampling-adjusted comparison with NGT (linear regression), cknown diabetes: nonfasting

Table 3. Univariate age-adjusted and sample design-based association of risk factors in men and women with undiagnosed diabetes mellitus compared to nondiabetic subjects aged 55 to 74 years: the KORA 2000 Survey, Augsburg, Germany

\begin{tabular}{|c|c|c|c|c|}
\hline \multirow[t]{2}{*}{ Variables } & \multicolumn{2}{|l|}{ Men } & \multicolumn{2}{|l|}{ Women } \\
\hline & Odds ratio ${ }^{\mathrm{a}}$ & $95 \% \mathrm{CI}$ & Odds ratio ${ }^{\mathrm{a}}$ & $95 \% \mathrm{CI}$ \\
\hline Waist circumference $(\mathrm{cm})^{\mathrm{b}}$ men: $>109$, women: $>100$ & 2.6 & $1.5-4.6$ & 1.9 & $1.04-3.5$ \\
\hline Blood pressure $>140 / 90 \mathrm{mmHg}$ & 1.8 & $1.2-2.8$ & 2.3 & $1.6-3.2$ \\
\hline Triglycerides $\geq 2.0 \mathrm{mmol} / 1$ & 2.2 & $1.4-3.4$ & 3.0 & $1.6-5.5$ \\
\hline Parental diabetes history (yes) & 2.0 & $1.3-3.1$ & 2.0 & $1.04-3.8$ \\
\hline Low leisure time physical activity & 1.6 & $0.9-2.9$ & 1.6 & $0.96-2.6$ \\
\hline Hospitalization last 12 months & 1.4 & $0.8-2.5$ & 2.3 & $0.7-6.9$ \\
\hline Physician visit last 4 weeks & 0.7 & $0.4-1.2$ & 0.7 & $0.4-1.2$ \\
\hline
\end{tabular}

a Odds ratio (95\% confidence interval): sampling-adjusted logistic regression models including age-classes, using nondiabetic subjects as reference category (IGT, IFG and NGT)

b Waist circumference above 80th sex-specific centile

triglyceridaemia, and parental diabetes history in both sexes in univariate analyses (Table 3 ). Obesity was related to undiagnosed diabetes in men $(p<0.05)$. Hyperuricaemia, low leisure time physical activity, and health care utilization were not related in both sexes.

In multivariate analyses all associated risk factors from univariate analyses and age (classes) were included (Table 3). As BMI and waist circumference were highly correlated $(\mathrm{r}=0.78 ; p<0.001)$, only the latter was used in the multivariate model. In men, increased waist
Hypertensive blood pressure was defined by systolic blood pressure more than $140 \mathrm{~mm} \mathrm{Hg}$ or diastolic blood pressure more than $90 \mathrm{~mm} \mathrm{Hg}$ (with or without antihypertensive medications)

circumference (OR; 95\%CI: 2.3; 1.3-4.1), hypertensive blood pressure $(1.7 ; 1.1-2.6)$ and parental history $(1.9 ; 1.2-2.9)$ were related to undiagnosed diabetes.

In women, hypertriglyceridaemia (OR; 95\%CI: 2.7; 1.4-5.3), hypertensive blood pressure $(2.1 ; 1.4-3.0)$ and parental diabetes history $(2.0 ; 1.1-3.5)$ were associated with unknown diabetes in multivariate analysis (Table 3).

The NNTS $(95 \% \mathrm{CI})$ to identify one person with undiagnosed diabetes for the various risk factor groups 
Table 4. Sample design-based number needed to screen (NNTS) for undiagnosed diabetes in various risk factor groups in the population aged 55 to 74 years: the KORA 2000 Survey, Augsburg, Germany

\begin{tabular}{|c|c|c|c|c|}
\hline \multirow[t]{2}{*}{ Variables } & \multicolumn{2}{|l|}{ Men } & \multicolumn{2}{|l|}{ Women } \\
\hline & $\mathrm{NNTS}^{\mathrm{a}}$ & $95 \% \mathrm{CI}$ & NNTSa & $95 \% \mathrm{CI}$ \\
\hline Waist circumference $(\mathrm{cm})^{\mathrm{b}}$ men: $>109$, women: $>100$ & 5.1 & $3.4-7.9$ & 8.5 & $5.5-13.7$ \\
\hline Blood pressure $>140 / 90 \mathrm{mmHg}$ & 7.3 & $5.3-10.2$ & 8.5 & $6.2-11.9$ \\
\hline Triglycerides $\geq 2.0 \mathrm{mmol} / 1$ & 5.9 & $4.6-7.6$ & 6.2 & $4.1-9.6$ \\
\hline Parental diabetes history (yes) & 6.1 & $4.4-8.6$ & 8.8 & $5.8-13.8$ \\
\hline Low leisure time physical activity & 8.1 & $5.7-11.8$ & 11.3 & $8.1-16.0$ \\
\hline Hospitalization last 12 months & 7.5 & $4.7-12.5$ & 7.4 & $3.7-16.2$ \\
\hline Physician visit last 4 weeks & 11.6 & $7.6-18.0$ & 16.3 & $11.6-23.1$ \\
\hline
\end{tabular}

a Number needed to screen (95\% confidence interval): sampling-adjusted logistic regression models

b Waist circumference above 80th sex-specific centile

are shown in Table 4. In men, the lowest NNTS were found for individuals with low HDL-cholesterol, increased waist circumference, and hypertriglyceridaemia. In women, NNTS were lowest in subjects with hypertriglyceridaemia, individuals with low HDL-cholesterol and with previous hospitalization.

Taking into account the results of the multivariate regression analyses, a combination of abdominal adiposity, hypertension, and parental diabetes history in men resulted in a NNTS of 2.9 (95\%CI: 2.0-4.6). In women, the combination of increased triglycerides, hypertension, and parental history of diabetes yielded a NNTS of 3.2 (95\%CI: 2.2-5.1).

Telephone survey of non-participants. Of the non-participants 497 (50\%) could be reached by telephone interviews. The age-sex-characteristics of those who participated in the interview were not different from those who completely refused participation $(p \geq 0.05)$. The prevalence of self-reported diabetes mellitus among interviewed non-participants was higher compared to survey participants $(14.2 \%$ vs. $8.2 \%)$ $(p<0.001)$. Body mass index (BMI), based on self-reported height and weight, was lower among those who refused to participate (means \pm SD: $26.6 \pm 4.3$ vs $\left.28.6 \pm 4.4 \mathrm{~kg} / \mathrm{m}^{2}\right)(p<0.01)$. Fitting a multiple logistic regression model with non-participation in biomedical investigations as dependent variable, non-participants were less likely to be obese (BMI $\geq 30 \mathrm{~kg} / \mathrm{m}^{2}$ ) (OR; 95\%CI: $0.4 ; 0.3-0.6$ ), to be men (OR: 0.8; 0.6-0.97), and were more likely to be older than participants (OR: 65-74 vs 55-64 years: 1.4; 1.1-1.7). Furthermore, non-participants more often reported known diabetes mellitus (OR: 2.2; 1.6-3.0).
Hypertensive blood pressure was defined by systolic blood pressure more than $140 \mathrm{~mm} \mathrm{Hg}$ or diastolic blood pressure more than $90 \mathrm{~mm} \mathrm{Hg}$ (with or without antihypertensive medications)

\section{Discussion}

This is the first population-based study on prevalence of diabetes (diagnosed and undiagnosed) and IGT in an 55 to 74 year old population in Germany using OGTT. The total diabetes prevalence was about $17 \%$, an additional $23 \%$ of the population had IGT or IFG. Undiagnosed diabetes was much more frequent than previously expected, about half of the cases of diabetes were undiagnosed.

Comparable data from other regions in Germany on the prevalence of known and newly diagnosed diabetes is lacking. The age-sex-specific prevalences of self-reported diabetes in the 1998 German National Health Interview and Examination Survey (West-Germany) were slightly higher (about 1\%) as in the Augsburg study, which could be due to geographic variations and different assessment methods [5]. A low prevalence of $1 \%$ was estimated for undetected diabetes in the National Health Survey (age-group 18-79 years) (known diabetes: 4.7\%) [5]. However, undetected diabetes was defined as combinations of increased fasting glucose, $\mathrm{HbA}_{1 \mathrm{c}}$, and urine glucose, sensitivity and specificity of this approach were not given. In the RIAD (Risk factors in IGT for Atherosclerosis and Diabetes) study from Dresden, the OGTT-based prevalence of newly diagnosed diabetes (WHO criteria) in subjects aged 40 to 70 years with an increased risk for Type 2 diabetes was 15\% [17]. Thus, the RIAD Study and another $\mathrm{HbA}_{1 \mathrm{c}}$-based investigation confirm that undiagnosed diabetes is more common in the German population than previously thought $[17,18]$.

It has been hypothesized that undiagnosed diabetes in Germany is rare because of intensive health care utilization by the population [5]. However, the extent of health care use (physician visits, hospitalization) 
was not related to the risk of having undiagnosed diabetes [19]. About $40 \%$ of those in this study had at least one physician contact during the preceding 4 weeks, which was similar to people with normal glucose tolerance. Thus, lack of diabetes diagnosis seems not to be related to less utilization of health care.

The prevalence of abnormal glucose tolerance found in Southern Germany is among the highest reported from European countries [7, 20, 21, 22]. Obesity could be a main predictor for the high prevalence of diabetes and IGT in Germany. Data from the WHO MONICA project and other studies indicate a high percentage of obese and overweight subjects in the German population compared to other countries in Western and Middle Europe, in particular, among men $[23,24]$. Currently, about $20 \%$ of the German adult population can be considered obese $[12,23]$. In a longitudinal analysis of the MONICA Augsburg surveys from 1989/90 to 1994/95, even a constant temporal increase was observed both for BMI and waist circumference [12]. Given the high prevalence of diabetes and glucose intolerance found in this study and the large impact of obesity on incident diabetes observed in a follow-up of the Augsburg MONICA project [25], prevention and management of obesity is of great importance for public health in Germany.

When applying the Augsburg estimates to the total German population, there could be currently about 1.5 million individuals with undiagnosed diabetes in the age-group 55 to 74 years. Furthermore, about 3 million in this age-group have IGT, of whom a substantial number will develop diabetes within the next years. In absolute numbers, subjects with undiagnosed diabetes in the German population (55-74 years) already establish a larger group compared to patients with known Type 2 diabetes in the total population of other European countries like France (1.3 million) and the UK (1.2 million) [26]. Therefore, given the high diabetes prevalence and the large population in Germany, these epidemiologic results are also relevant from a European public health perspective.

Undiagnosed diabetes is not a mild disorder compared to clinically detected diabetes. Although subjects with undiagnosed diabetes were not as hyperglycaemic as those with known diabetes $\left(\mathrm{HbA}_{1 \mathrm{c}}\right)$, they have a high prevalence of risk factors for cardiovascular complications [27]. It is also noteworthy, that the levels of obesity, increased blood pressure and dyslipidemia found in patients with known and treated diabetes are similar to the high levels observed in newly diagnosed and therefore untreated diabetic subjects. Although the benefits of early detection and treatment of undiagnosed diabetes still need to be established [28], this study adds more evidence to justify opportunistic diabetes screening in subjects with known cardiovascular risk factors.

There were some sex differences in the possible target groups for diabetes screening. In men, screening could be most efficient in individuals with a combination of abdominal adiposity, hypertension and parental diabetes history. In women, a low NNTS was found in individuals with hypertriglyceridaemia, hypertensive blood pressure and parental diabetes. Overall, screening might be more efficient in men than women (lower NNTS). Data from the Health, Aging, and Body Composition Study from the United States confirm that screening for undiagnosed diabetes could be most efficient in subjects with increased cardiovascular risk factors, including obesity (abdominal adiposity) and history of hypertension, in particular, among men [16]. Increased triglycerides, which were associated with undiagnosed diabetes in women, are a simple marker for insulin resistance in the general population [29].

The ADA recommended the use of fasting glucose for diabetes screening, which could be obtained during routine primary care visits $[27,30]$. Overall, about $60 \%$ of the cases could have been diagnosed by fasting glucose in this study. However, in women, half of the subjects with newly diagnosed diabetes were only detected by 2 -h glucose (men: $34 \%$ ), confirming previously reported sex differences in the WHO and ADA diabetes diagnostic criteria [31]. Thus, there may not only be some sex differences in the "who" but also in the "how" for diabetes screening.

Several limitations of this study need to be considered. Despite enormous efforts only $62 \%$ of the subjects in the age-group 55 to 74 years agreed to participate, which was in the range of other recent population-based surveys in Germany and Europe [32]. To evaluate possible bias, we investigated the non-participants with respect to known diabetes and self-reported BMI. In general, non-participants in risk factor surveys are less healthy [33]. This 'healthy participant effect' was also observed in the KORA Survey 2000. Non-participants were about two times more likely to have diabetes mellitus. Therefore, the prevalence of known diabetes mellitus could have been underestimated. Obesity as a major risk factor for Type 2 diabetes mellitus was more frequently found among survey participants than in non-participants. This might have resulted in overestimating the prevalence of newly diagnosed diabetes and IGT, non-participants were also older. However, this bias was partly controlled, because the data has been weighted in the analysis to match the age-sex-distribution of the residential population in Augsburg.

In conclusion, about $40 \%$ of the population 55 to 74 years in the Augsburg region had disturbed glucose tolerance or diabetes. Half of the total cases with diabetes were undiagnosed. Cardiovascular risk factors worsen among glucose tolerance categories, justifying opportunistic diabetes screening. Screening for undiagnosed diabetes might be most efficient in individuals with abdominal adiposity (men), hypertriglyceridaemia (women), hypertensive blood pressure, and parental history of diabetes. 
Acknowledgements. The OGTT study was partly funded by the German Federal Ministry of Health, the Ministry of School, Science and Research of the State of North-Rhine-Westfalia, and the Anna Wunderlich-Ernst Jühling Foundation (WR, GG). The KORA Survey 2000 was financed by the GSF, which is funded by the German Federal Ministry of Education, Science, Research and Technology and the State of Bavaria. The authors are indebted to K. Papke (head of the KORA study centre) and B. Schwertner (survey organization) and their co-workers for organizing and conducting the data collection. We are grateful to Prof. H.E. Wichmann and Prof. van Eimeren (GSF, Neuherberg) for initiating the KORA Survey 2000. We also thank all participants of the OGTT study.

\section{References}

1. King H, Aubert RE, Herman WH (1998) Global burden of diabetes, 1995-2025: prevalence, numerical estimates, and projections. Diabetes Care 21:1414-1431

2. Haffner SM, Lehto S, Rönnemaa T, Pyörälä K, Laakso M (1998) Mortality from coronary heart disease in subjects with type 2 diabetes and in nondiabetic subjects with and without prior myocardial infarction. N Engl J Med 339: 229-234

3. Balkau B (2000) The DECODE Study. Diabetes epidemiology: collaborative analysis of diagnostic criteria in Europe. Diabetes Metab 26:282-286

4. The EURODIAB ACE Study Group (2000) Variation and trends in incidence of childhood diabetes in Europe. Lancet 355:873-876

5. Thefeld W (1999) Prevalence of diabetes mellitus among adults in Germany (in German). Gesundheitswesen 61[Suppl 2]: S85-S89

6. Löwel H, Stieber J, Koenig W et al. (1999) The diabetesrelated risk of myocardial infarction in a southern German population: results of the MONICA Augsburg studies 1985-1994 (in German). Diab Stoffw 8:11-21

7. Tuomilehto J, Korhonen HJ, Kartovaara L et al. (1991) Prevalence of diabetes mellitus and impaired glucose tolerance in the middle-aged population of three areas in Finland. Int J Epidemiol 20:1010-1017

8. Keil U, Kuulasmaa K (1989) WHO MONICA project: risk factors. Int J Epidemiol 18 [Suppl 1]:S46-S55

9. Chambless L, Cairns V, Herbold M et al. (1986) MONICAAugsburg. Survey sampling. GSF-Report 31/86, GSF, Neuherberg

10. World Health Organisation (1999) Definition, diagnosis and classification of diabetes mellitus and its complications. Part 1: Diagnosis and classification of diabetes mellitus. Report of a WHO consultation. WHO, Geneva

11. Expert Committee on the diagnosis and classification of diabetes mellitus (1997) Report. Diabetes Care 20:1183-1197

12. Liese AD, Döring A, Hense HW, Keil U (2001) Five year changes in waist circumference, body mass index and obesity in Augsburg, Germany. Eur J Nutr 40:282-288

13. Särndal CE, Swensson B, Wretman J (1992) Model assisted survey sampling. Springer-Verlag, New York

14. Lemeshow S, Cook ED (1999) Practical considerations in the analysis of complex sample survey data. Rev Epidemiol Sante Publique 47:479-487

15. Gleason J (1997) Computing intraclass correlations and large ANOVAs. Stata Technical Bulletin 35:25-31

16. Franse LV, Di Bari M, Shorr RI et al. (2001) Type 2 diabetes in older well-functioning people: who is undiagnosed?
Data from the Health, Aging, and Body Composition Study. Diabetes Care 24:2065-2070

17. Koehler C, Temelkova-Kurktschiev T, Schaper F, Fücker K, Hanefeld M (1999) Prevalence of newly detected type 2 diabetes, impaired glucose tolerance and impaired fasting glucose in a risk population. Data from the RIAD Study after implementation of the new diagnostic criteria for diabetes (in German). Dtsch med Wochenschr 124:1057-1061

18. Hauner H, Kurnaz AA, Haastert B, Groschopp C, Feldhoff KH (2001) Undiagnosed diabetes mellitus and metabolic control assessed by $\mathrm{HbA}(1 \mathrm{c})$ among residents of nursing homes. Exp Clin Endocrinol Diabetes 109:326-329

19. Young TK, Mustard CA (2001) Undiagnosed diabetes: does it matter? CMAJ 164:24-28

20. DECODE Study Group on behalf of the European Diabetes Epidemiology Study Group (1998) Will new diagnostic criteria for diabetes mellitus change phenotype of patients with diabetes? Reanalysis of European epidemiological data. BMJ 317:371-375

21. Mooy JM, Grootenhuis PA, de Vries H et al. (1995) Prevalence and determinants of glucose intolerance in a Dutch caucasian population. The Hoorn Study. Diabetes Care 18:1270-1273

22. Stolk RP, Pols HAP, Lamberts SWJ et al. (1997) Diabetes mellitus, impaired glucose tolerance, and hyperinsulinemia in an elderly population. The Rotterdam Study. Am J Epidemiol 145:24-32

23. Seidell JC (1995) Obesity in Europe. Obes Res 3 [Suppl 2]:89S-93S

24. Kuulasma K, Tunstall-Pedoe H, Dobson A et al. (2000) Estimation of contribution of changes in classic risk factors to trends in coronary-event rates across the WHO MONICA Project populations. Lancet 355:675-687

25. Meisinger C, Thorand B, Schneider A, Stieber J, Döring A, Löwel H (2002) Sex differences in risk factors for incident type 2 diabetes mellitus. The MONICA Augsburg cohort study. Arch Intern Med 162:82-89

26. Jönsson B (2002) Revealing the cost of Type II diabetes in Europe. Diabetologia 45:S5-S12

27. Harris MI, Eastman RC (2000) Early detection of undiagnosed diabetes mellitus: a US perspective. Diabetes Metab Res Rev 16:230-236

28. Wareham NJ, Griffin SJ (2001) Should we screen for type 2 diabetes? Evaluation against National Screening Committee criteria. BMJ 322:986-988

29. McAuley KA, Williams SM, Mann JI et al. (2001) Diagnosing insulin resistance in the general population. Diabetes Care 24:460-464

30. Lawrence JM, Bennett P, Young A, Robinson AM (2001) Screening for diabetes in general practice: cross sectional population study. BMJ 323:548-551

31. Pomerleau J, McKeigue PM, Chaturvedi N (1999) Relationship of fasting and postload glucose levels to sex and alcohol consumption. Are American Diabetes Association criteria biased against detection in women? Diabetes Care 22:430-433

32. Thefeld W, Stolzenberg H, Bellach BM (1999) German National Health Interview and Examination Survey: response, composition of participants, and analysis of nonrespondents (in German). Gesundheitswesen [Suppl 2]: S57-S61

33. Kleges RC, Williamson JE, Somes GW, Talcott GW, Lando HA, Haddock CK (1999) A population comparison of participants and nonparticipants in a health survey. Am J Public Health 89:1228-1231 